\title{
A STUDY OF THE INFLUENCE OF IRRADIATION OF CARBON DIAMOND-LIKE COATINGS WITH NANOSECOND LASER PULSES ON THEIR STRUCTURAL-PHASE COMPOSITION AND TRIBOLOGICAL PROPERTIES
}

\author{
Yu. R. Kolobov, ${ }^{1}$ S. S. Manokhin, ${ }^{1}$ A. Ya. Kolpakov, ${ }^{2}$ \\ A. I. Poplavskii, ${ }^{2,3}$ M. N. Yapryntsev ${ }^{2}$ G. V. Odintsova, ${ }^{4}$ \\ V. I. Betekhtin, ${ }^{5}$ A. G. Kadomtsev, ${ }^{5}$ and M. V. Narykova ${ }^{5}$
}

UDC 539.211:544.015: 538.93:538.95

\begin{abstract}
Using the methods of Raman spectroscopy, optical metallography and scanning electron microscopy, combined with the measurements of tribological characteristics, special aspects of the formation of microrelief and variation of the structural-phase state of carbon coatings formed on the surface of AISI 321 heat-resistant steel by pulsed cathodic arc deposition followed by their irradiation with nanosecond laser pulses are studied.
\end{abstract}

Keywords: solid amorphous carbon films, nanosecond laser modification, friction and wear.

\section{INTRODUCTION}

The formation of carbon coatings, whose structure, relief and phase composition can be controlled, generates a great interest in view of a possibility of improving their physical-mechanical properties and increasing the corrosion and wear resistance, as well as biological and other properties of various materials [1, 2]. Such coatings are widely used as protective films of hard discs, magnetic heads and cantilevers for scanning probe microscopes due to their high hardness, chemical inertness and insulating properties close to those of diamonds (diamond-like coatings) [3].

One of the promising research directions in the field of the development of new technologies for processing medical materials is their surface modification with concentrated energy fluxes, in particular, with short and ultrashort laser radiation pulses [4]. As a result of this treatment, the near-surface layers of materials can be modified to a depth comparable with the laser beam diameter (tens and hundreds of micrometers). The processes excited by this treatment, which are associated with physical-chemical transformations and surface topography changes, make it possible to form a unique complex of physical-chemical and mechanical properties in the surface, near-surface and sub-surface layers of the materials. By varying the laser radiation parameters, we can under control change the surface relief from the macroto a nano-level, among other things, creating multiscale roughness. A transition from the mode of material ablation to a mode of shock-wave treatment allows improving the mechanical properties of near-surface layers of the material without substantial changes of the surface topography. These structures can affect the material characteristics important for their practical use (such as fatigue strength, wettability, and wear and corrosion resistance of the near-surface layer). An analysis of the literature data demonstrates that this research avenue remains to be important and promising at

${ }^{1}$ Institute of Problems of Chemical Physics of the Russian Academy of Sciences, Chernogolovka, Russia, e-mail: kolobov@icp.ac.ru; manohin@bk.ru; ${ }^{2}$ National Research University "Belgorod State University" (BelSU), Belgorod, Russia, e-mail: kolpakov@bsu.edu.ru; yaprintsev@bsu.edu.ru; ${ }^{3}$ V. G. Shukhov Belgorod State Technological University, Belgorod, Russia, e-mail: poplavsky@bsu.edu.ru; ${ }^{4}$ ITMO University, St. Petersburg, Russia, e-mail: gvodintsova@corp.ifmo.ru; ${ }^{5}$ Ioffe Institute, St. Petersburg, Russia, e-mail: Vladimir.betekhtin@mail.ioffe.ru; Andrej.Kadomtsev@mail.ioffe.ru; Maria.Narykova@mail.ioffe.ru. Translated from Izvestiya Vysshikh Uchebnykh Zavedenii, Fizika, No. 6, pp. 84-88, June, 2021. Original article submitted December 2, 2020. 
present. For instance, there are well-known methods of laser (optical) forging, due to which it is possible, along with the method of surface microstructuring (designing multiscale surface roughness aimed at extreme wettability characteristics), to nanostructure thin near-surface layers of metallic materials [5]. At present (according to the data reported by Jiu et al. [6] and $\mathrm{Lu}$ et al [7]), it is possible to achieve material nanostructuring to a depth of $0.5 \mathrm{~mm}$ and more. This research direction has been reported for the recent two decades by a team of co-authors $[8,9]$.

There are single data available in the literature on the application of lasers for texturing thin diamond-like coatings, optimization of tribological properties [10-12], and laser-induced graphitizing of diamond-like films [13]. Dumitru et al. [14] report the information on the influence of this treatment on the friction coefficient. They showed that laser texturing, consisting of shallow depressions ( $25 \mu \mathrm{m}$ in diameter and $15-20 \mu \mathrm{m}$ in depth), had considerably increased the service life of the diamond-like coating. Unfortunately the information available in the current literature is insufficient for getting an insight into the laser influence on the tribological properties of diamond-like coatings deposited on metallic substrates, since most of the data concern the diamond-like coatings formed on silicon substrates. The laser irradiation modes known from the literature to a large extent differ from those used in the present study. In this connection, it is challenging to clarify the influence of short-pulse irradiation of carbon coatings deposited on the surface on the variation of their microstructure and phase compositions. Using carbon coatings formed on steel substrates as an example, we study the action of nanosecond laser pulses on the changes of their microstructure and phase compositions in order to identify the influence of these changes on tribological properties of these coatings.

\section{EXPERIMENTAL MATERIALS AND METHODS}

In this work we studied the influence of nanosecond laser radiation pulses on carbon coatings formed on the substrates of AISI 321 heat-resistant steel and single-crystal silicon (model material). The coatings were formed by the method of pulsed cathodic arc deposition (PCAD). Nearly $1 \mu \mathrm{m}$ thick carbon coatings were deposited in vacuum on the polished steel substrates. Before deposition, the vacuum chamber was pumped off to a pressure of $4 \cdot 10^{-3} \mathrm{~Pa}$ and the surface was cleaned by argon ions using an ion source in the following operating modes: discharge voltage $-2.0 \mathrm{kV}$, discharge current $-100 \mathrm{~mA}$, argon pressure $-0.5 \mathrm{~Pa}$, negative bias voltage on the substrate $-2.0 \mathrm{kV}$, cleaning time -10 min. The technological parameters of the PCAD process were as follows: vacuum chamber pressure $-8 \cdot 10^{-3} \mathrm{~Pa}$, discharge pulse amplitude $-1 \mathrm{kA}$, pulse duration $-1.2 \mathrm{~ms}$, discharge voltage $-300 \mathrm{~V}$, pulse repetition frequency -3 $\mathrm{Hz}$, no negative voltage was supplied to the substrate (floating potential), deposition rate $-0.18 \mathrm{~nm} / \mathrm{pulse}(33 \mathrm{~nm} / \mathrm{min}$ at the pulse repetition frequency of $3 \mathrm{~Hz}$ ). The substrate temperature did not exceed $70^{\circ} \mathrm{C}$.

The resulting samples were then treated with nanosecond laser pulses irradiating the entire sample area at the energy density of $F_{0}=2.66 \mathrm{~J} / \mathrm{cm}^{2}$. For irradiation, an ytterbium fiber laser was used with a pulse duration of $\tau=100 \mathrm{~ns}$ and a wavelength of $\lambda=1.06 \mu \mathrm{m}$. The pulse repetition frequency was $f=60 \mathrm{kHz}$. The sample surface was irradiated by consecutive scanning with a focused laser beam with a diameter of $d_{0}=50 \mu \mathrm{m}$. After one scanning row at the pulse repetition frequency $f$ at a scanning step of $M_{x}$ is completed, the beam is displaced along the $Y$ axis to the next row where the scanning step is $M_{y}$. This process is described in greater detail elsewhere $[15,16]$.

The comprehensive tribological examination of the samples included a continuous recording of the friction coefficient to an accuracy of \pm 0.01 during attrition tests in accordance with a standard ball-on-disk scheme (ASTM G99-959 and DIN 50324) in an automated friction testing machine (Tribometer, CSM Instruments, USA), and a fractographic analysis of the wear tracks on the coating and wear scars on the counterbody $\left(6 \mathrm{~mm}\right.$ diameter $\mathrm{Al}_{2} \mathrm{O}_{3}$ ball). The tests were carried out in air at a load of $1 \mathrm{~N}$, a linear velocity of $10 \mathrm{~cm} / \mathrm{s}$, a wear circle radius of $6.5 \mathrm{~mm}$, and a rubbing path of $300 \mathrm{~m}$. After testing, the appearance of wear traces on the coatings and wear scars on the ball were examined with an Olympus GX 51 inverted metallurgical microscope. The average diameter of the wear scars was measured to an accuracy of no worse than $\pm 0.01 \mu \mathrm{m}$. The average values of the wear track cross sectional area and depth were performed in four diametrally and orthogonally opposite regions of the sample using a Surtronic 25 precision profilometer (Taylor Hobson) to a measurement accuracy of $\pm 0.01 \mu \mathrm{m}$. The quantitative estimation of wear resistance of the samples and the counterbody was performed from the wear factor value $W$ [17], its calculation procedure is detailed in [18]. 
TABLE 1. Tribological Properties of Samples in Different States

\begin{tabular}{l|c|c}
\hline \hline \multicolumn{1}{c|}{ Samples } & $\begin{array}{c}\text { Friction coefficient } \\
\text { during testing, } \mu\end{array}$ & Sample wear factor $\left(\times 10^{-7}\right), \mathrm{mm}^{3} \cdot \mathrm{H}^{-1} \cdot \mathrm{m}^{-1}$ \\
\hline Initial steel, uncoated & 0.81 & 7444 \\
\hline Laser-irradiated steel, uncoated & 0.89 & 3298 \\
\hline Steel with a carbon diamond-like coating & 0.12 & 2.783 \\
\hline $\begin{array}{l}\text { Steel with a carbon coating irradiated with } \\
\text { a nanosecond laser }\end{array}$ & $\mathbf{0 . 7 5}$ & $\mathbf{0 . 6 6}$ \\
\hline
\end{tabular}

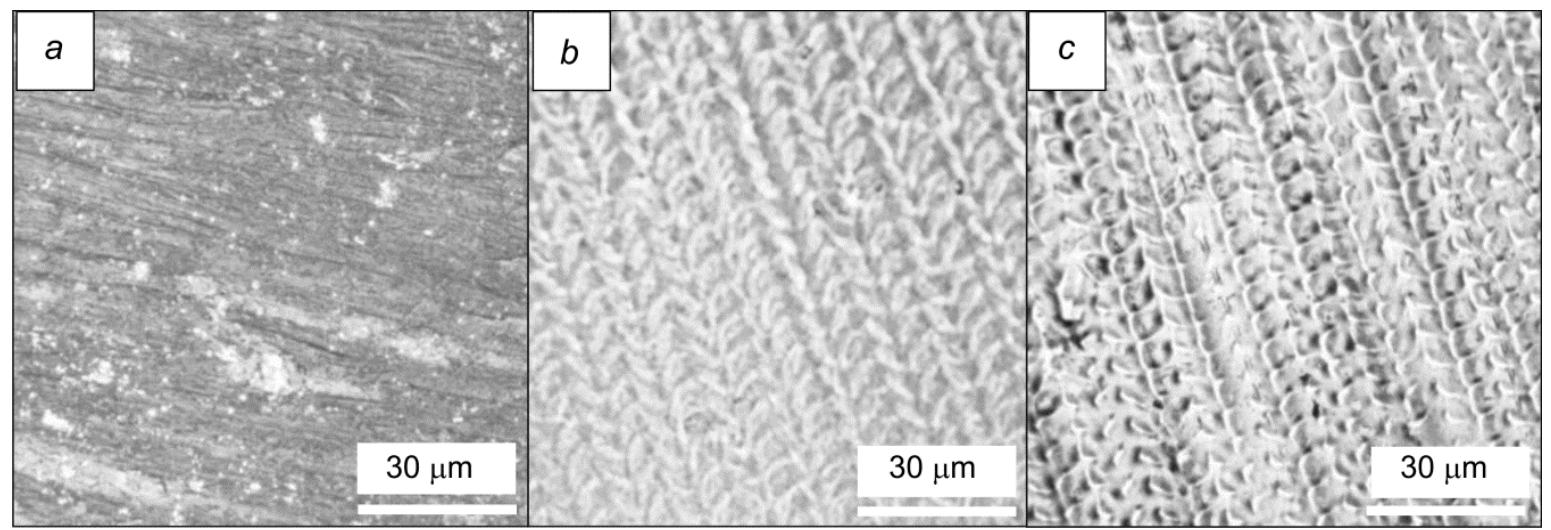

Fig. 1. Surface structure of the AISI 321 steel samples: $a$ - carbon coating in the initial state, $b-$ initial surface (without coating) irradiated with nanosecond laser pulses. Optical metallography.

The surface morphology and the structure of the modified near-surface layers were examined using a FEI Quanta 600 scanning electron microscope (FEI, Japan) with a field emission gun (cathode) and an Altami metallographic microscope (Altami, Russia).

The structural studies were carried out using Raman spectroscopy of light in a LabRAM HR Evolution Raman spectrometer at the laser radiation length of $532 \mathrm{~nm}$. The spectra were analyzed from the positions of D- and G peaks in accordance with the model describing three stages of carbon material structure changes [19]: ideal graphite $\rightarrow$ nanocrystalline graphite $(\mathrm{nc}-\mathrm{G}) \rightarrow$ amorphous graphite $(\mathrm{a}-\mathrm{C}) \rightarrow$ tetrahedral amorphous carbon $(\mathrm{ta}-\mathrm{C})$. The spectra were analyzed using the positions of $\mathrm{D}$ and $\mathrm{G}$ peaks, their peak intensity ratio $I(\mathrm{D}) / I(\mathrm{G})$, and from the peak width at half height calculated as the difference between the maximum and minimum values of the function argument taken at the level equal to its half maximum value. The $G$ and $D$ peaks were described using the following combination: a BreitWigner-Fano line shape for $\mathrm{G}$ peak and a Lorentzian for $\mathrm{D}$ peak.

\section{EXPERIMENTAL RESULTS AND DISCUSSION}

In order to study the peculiarities of the structural-phase state and properties of the modified coatings, a special mode of laser irradiation was selected, which, according to the authors, causes a minimum damage of the carbon coating.

As an example, Fig. 1 presents the surface microstructure images after different trteatment modes, which were obtained by the method of optical metallography. Such laser-induced quasi-periodic surface structures are well known for their unique optical, antibacterial, biological and hydrophobic properties.

We have determined the tribological properties (wear factor, friction coefficient) of the above-described samples in different states. The values of wear and friction coefficients are listed in Table 1. 


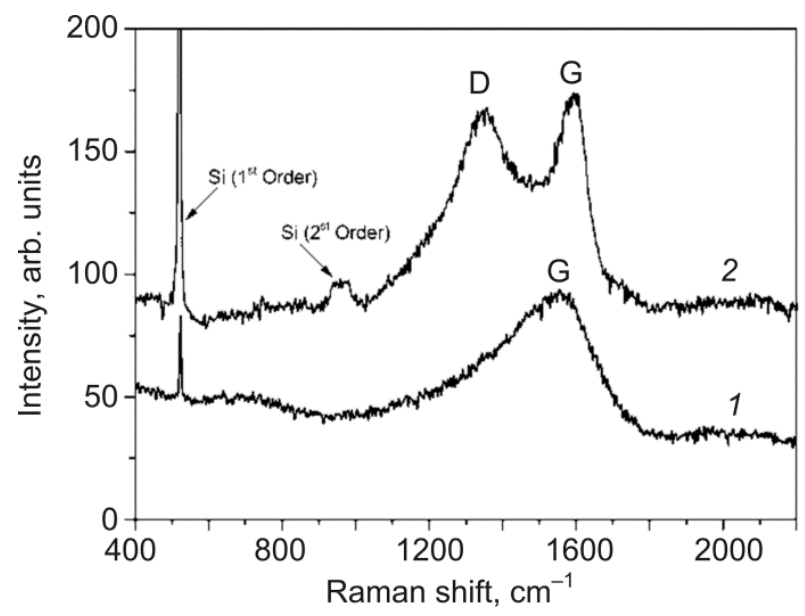

Fig. 2. Spectrum from the sample with a carbon coating formed by the PCAD method, before (Curve 1) and after (Curve 2) laser irradiation.

The data in Table 1, obtained from the profilograms of the wear tracks, clearly show that the wear of the sample surface with a carbon coating on the AISI 321 steel substrate irradiated with a nanosecond laser occurs by a factor of four less intensively compared to the nonirradiated sample having a carbon diamond-like coating. However, compared to the initial surface state (without coating, without irradiation) the wear factor is a few orders of magnitude lower. It should be noted that the friction coefficient of the samples (with a preliminary deposited coating) considerably increases compared to that of the sample not subjected to laser irradiation and nearly corresponds to the initial state level. It is very likely that the friction coefficient increases due to the increased sample surface roughness after additional laser irradiation.

The changes of the structural-phase states of carbon coatings taking place as a result of the impact of nanosecond laser pulses were studied (using the methods of Raman and scanning electron spectroscopy) on the model reference samples with carbon coatings deposited on the substrates cut from the plates of single crystal silicon. This model material was selected for the substrates in order to rule out the effect of the steel substrate on the results of examination of the structural-phase composition of the carbon coating.

Figure 2 presents the spectra from the samples with a coating formed by the PCAD method before (Spectrum 1) and after (Spectrum 2) laser irradiation. The spectrum from the initial sample has a single prominent wide G-peak, characteristic for amorphous carbon. Note that D-peak is not seen clearly. After laser irradiation, there is a clearly seen D-peak in the Raman spectrum, i.e., the initial amorphous state of the carbon coating has partially transformed into disordered nanocrystalline graphite. This is likely to be the reason while the tribological properties have changed, as it was shown above.

The initial sample structure represents a mixture of amorphous carbon and carbon with tetrahedral atomic arrangement. This conclusion relies on the G-peak position.

Therefore, the structure of the laser-irradiated coating represents an intermediate state between nanocrystalline graphite and amorphous carbon. The fraction of $s p^{2}$-phase in it accounts for more than $80 \%$, and the diameter of $s p^{2}$ clusters consisting of hexagonal rings is about $1.3 \mathrm{~nm}$.

\section{CONCLUSIONS}

It has been shown that laser irradiation allows changing the structural-phase state of carbon diamond-like coatings. Using a diamond-like coating on the AISI 321 heat-resistant steel, it has been demonstrated that an additional laser irradiation of the surface improves the wear characteristics of the coating by nearly an order of magnitude 
compared to conventional surface treatment by the methods of pulsed cathodic arc deposition. It has been found out that this effect is related to the change of the structural-phase composition of the carbon coating, formed by the PCAD method, as a result of laser irradiation. The initial carbon coating structure is X-ray amorphous, and in the samples subjected to laser irradiation reperesents an intermediate state between nanocistalline graphite and amorphous carbon. Under the condition of nanosecond laser irradiation, a phase transformation is taking place in the diamond-like coating accompanied by substantial changes of the volume fractions of $s p^{3}$ - and $s p^{2}$-bonds.

The carbon coating formed by the PCAD method, followed by its modification with laser radiation, can be used for forming a relief with predetermined surface roughness aimed at increasing the wear of materials for engineering applications and for improving the biocompatibility of such medical materials as titanium and its alloys.

This study in its main part, dealing with a study of mechanical properties of coated alloys, has been supported by the RSF grant No. 19-12-00221 and in its additional part, reporting a study of the structural-phase compositions of the coatings, has been supported by the Theme map for the IPCP RAS in accordance with the theme of the government assignment, the state registration number AAAA-A19-119100800130-0. The research work was carried out using the scientific equipment of the SC "Technologies and materials" of the NRU BelSU (Belgorod).

\section{REFERENCES}

1. Yu. R. Kolobov, Nanotechnologies in Russia, 4, No.11-12, 69 (2009); http://link.springer.com/journal/12201/ volumes-andissues/4-11; http://link.springer.com/article/10.1134/S1995078009110020.

2. R. A. Andrievskii, Physics. Uspekhi, 184, Iss. 10, 1017 (2014).

3. M. Yoshikawa, G. Katagiri, H. Ishida, et al., Appl. Phys. Lett., 52, No. 19, 1639 (1988).

4. V. V. Stolarov, G. Zh. Sakhvadze, and Yu. V. Baranov, Konstruktsii Composit. Mater., No. 3, 13 (2007).

5. Yu. R. Kolobov, E. V. Golosov, T. N. Vershinina, et al., Appl. Phys. A. Mater. Sci. Proc., 119, No. 1, 241 (2015).

6. W. Jia, Q. Hong, H. Zhao, et al., Mater. Sci. Eng. A, 606, 354 (2014).

7. J. Z. Lu, L. J. Wu, G. F. Sun, et al., Acta Materialia, 127, 252 (2017).

8. Yu. R. Kolobov, Russ. Phys. J., 61, No. 4, 611 (2018); http://link.springer.com/article/10.1007/s11182-018$1440-4$.

9. N. Shchedrina, Y. Karlagina, T. E. Itina, et al., Opt. Quantum Electron., 52, No. 3, 1 (2020).

10. A. Erdemir, Tribology Int., 38, No. 3, 249 (2005).

11. K.A.H. Al Mahmud, M. A. Kalam, H. H. Masjuki, et al., Critical Rev. Solid State Mater. Sci., 40, No. 2, 90 (2015).

12. A. R. Konicek, D. S. Grierson, P.U.P.A. Gilbert, et al., Phys. Rev. Lett., 100, No. 23, 235502 (2008).

13. L. C. Nistor, J. Van Landuyt, V. G. Ralchenko, et al., Appl. Phys. A, 58, No. 2, 137 (1994).

14. G. Dumitru, V. Romano, H. P. Weber, et al., Diamond Related Mater., 12, No. 3-7, 1034 (2003).

15. E. I. Ageev, Y. M. Andreeva, Y. Y. Karlagina, et al., Laser Phys., 27, No. 4, 046001 (2017).

16. V. P. Veiko, G. V. Odintsova, M. Y. Gazizova, et al., Laser Phys., 28, No. 8, 086002 (2018).

17. D. S. Vershinin, M. Yu. Smolyakova, S. S. Manokhin, et al., Prom. Lab. Diagnost. Mater., 76, No. 12, 45 (2010).

18. M. G. Kovaleva, A. J. Kolpakov, A. I. Poplavsky, and M. E. Galkina, J. Friction and Wear, 34, No. 6, 481 (2013).

19. A. C. Ferrari and J. Robertson, Phys. Rev. Lett., 97, No. 18, 187401 (2006). 